\title{
Association between anti-ZnT8 autoantibody specificities and $S L C 30 A 8$ Arg325Trp variant in Japanese patients with type 1 diabetes
}

\author{
E. Kawasaki • M. Uga • K. Nakamura • G. Kuriya • \\ T. Satoh • K. Fujishima - M. Ozaki • N. Abiru • \\ H. Yamasaki • J. M. Wenzlau • H. W. Davidson • \\ J. C. Hutton • K. Eguchi
}

Received: 9 July 2008 / Accepted: 1 September 2008 / Published online: 11 October 2008

(C) Springer-Verlag 2008

\begin{abstract}
Aims/hypothesis We analysed the association between humoral autoreactivity to zinc transporter-8 (ZnT8) and the SLC30A8 rs13266634 polymorphism (Arg325Trp), which is located at the most distal loop in the ZnT8 protein. Methods Autoantibodies to ZnT8 were determined by RIA in 270 patients with type 1 diabetes using ZnT8 carboxyterminal constructs (amino acids 268-369) carrying 325Trp (CW) and $325 \operatorname{Arg}(\mathrm{CR})$ and a hybrid construct (CW-CR). Forty-four ZnT8 autoantibody-positive sera with genomic DNA were used to examine the association between reactivity to ZnT8 constructs and the rs13266634 genotype. Results Seventy-five patients reacted to the CW-CR hybrid construct, whereas 37 and 36 patients reacted to the CW and CR constructs, respectively. All sera positive for either
\end{abstract}

E. Kawasaki $(\bowtie) \cdot$ M. Uga $\cdot$ K. Nakamura $\cdot$ G. Kuriya

Department of Metabolism/Diabetes and Clinical Nutrition,

Nagasaki University Hospital of Medicine and Dentistry,

1-7-1 Sakamoto,

Nagasaki 852-8501, Japan

e-mail: eijikawa@nagasaki-u.ac.jp

T. Satoh $\cdot$ K. Fujishima $\cdot$ N. Abiru $\cdot$ K. Eguchi

Department of Endocrinology and Metabolism,

Unit of Translational Medicine,

Nagasaki University Graduate School of Biomedical Sciences,

Nagasaki, Japan

M. Ozaki $\cdot$ H. Yamasaki

Center for Health and Communicating Medicine,

Nagasaki University,

Nagasaki, Japan

J. M. Wenzlau $\cdot$ H. W. Davidson · J. C. Hutton

Barbara Davis Center for Childhood Diabetes,

University of Colorado at Denver and Health Sciences Center,

Aurora, CO, USA
$\mathrm{CW}$ or $\mathrm{CR}$ autoantibodies were positive for CW-CR autoantibodies. Among 19 patients with a $325 \operatorname{Arg}(\mathrm{CC})$ genotype, $5 \%$ had $\mathrm{CW}$-specific autoantibodies, $42 \%$ had CR-specific autoantibodies and $32 \%$ had dual reactivity. Conversely, $73 \%$ of 15 patients with the $325 \operatorname{Trp}(\mathrm{TT})$ genotype had $\mathrm{CW}$-specific autoantibodies, no patients had CR-specific autoantibodies and 13\% had dual reactivity. Nine of the ten patients $(90 \%)$ with the CT genotype reacted with either $\mathrm{CR}$ or $\mathrm{CW}$ constructs. The titre of $\mathrm{CR}$ autoantibodies in patients carrying the $\mathrm{C}$ allele was significantly higher than that in TT homozygotes $(p<0.0001)$. In contrast, the titre of CW autoantibodies in patients carrying a $\mathrm{T}$ allele was significantly higher than that in CC homozygotes $(p<0.005)$. No evidence of an association between rs13266634 and type 1 diabetes was observed.

Conclusions/interpretation These results indicate that variant residue at amino acid 325 is a key determinant of humoral autoreactivity to ZnT8 and that the SLC30A8 genotype is an important determinant of autoantibody specificity.

Keywords Autoantibodies - Epitope - Genetics · Japanese population $\cdot S L C 30 A 8$. Type 1 diabetes $\cdot$ Zinc transporter-8

\begin{tabular}{|c|c|}
\hline \multicolumn{2}{|c|}{ Abbreviations } \\
\hline aa & amino acids \\
\hline CR & $\begin{array}{l}\text { zinc transporter-8 carboxy-terminal construct } \\
\text { carrying } 325 \mathrm{Arg}\end{array}$ \\
\hline $\mathrm{CW}$ & $\begin{array}{l}\text { zinc transporter-8 carboxy-terminal construct } \\
\text { carrying } 325 \operatorname{Trp}\end{array}$ \\
\hline $\begin{array}{l}\mathrm{CW}- \\
\mathrm{CR}\end{array}$ & $\begin{array}{l}\text { hybrid ZnT8 construct generated by fusion of } \\
\mathrm{CW} \text { and } \mathrm{CR}\end{array}$ \\
\hline
\end{tabular}


SNP single nucleotide polymorphism

ZnT8 zinc transporter-8

ZnT8A zinc transporter-8 autoantibodies

\section{Introduction}

Zinc transporters belonging to the SLC30 protein family are multipass transmembrane proteins with a role in the transport of zinc out of the cytoplasm or into the vesicles [1]. One of these, zinc transporter-8 (ZnT8), is specifically produced in the pancreatic beta cells and localised into insulin secretory granules and was recently identified as a major autoantigen in human type 1 diabetes [2, 3]. The gene coding for ZnT8 (solute carrier family 30 member 8; SLC30A8) is mapped to chromosome 8q24.11 [3]. The reference gene contains eight exons, spans $37 \mathrm{~kb}$, and encodes a 369 amino acid protein. In genome-wide association studies Sladek and colleagues reported an association between a non-synonymous variant in SLC30A8 (rs13266634; Arg325Trp) and susceptibility to type 2 diabetes [4]. The fact that major epitope(s) for ZnT8 autoantibodies (ZnT8A) lie within the cytoplasmic domain of the molecule (amino acids [aa] 268-369) [2] and the variant residue at aa325 is located at the most distal extension of the molecule into the cytoplasm [5] prompted us to analyse the association between humoral autoreactivity to ZnT8 and the SLC30A8 polymorphism.

\section{Methods}

Initially, sera from 270 Japanese patients with type 1 diabetes (61\% female), including 112 new-onset patients, were used to identify the ZnT8A-positive samples. The median age at onset and median duration of diabetes were 25.0 (range 1.0-77.0) years and 2.0 (range 0.0-42.0) years, respectively. From the total group, genomic DNA samples were available for 171 patients and were genotyped for the rs13266634 single nucleotide polymorphism (SNP). To examine the relationship between humoral autoreactivity to ZnT8 and the SLC30A8 variant, ZnT8A-positive patients with available genomic DNA samples were used. All patients met the revised criteria of the American Diabetes Association for type 1 diabetes [6]. Furthermore, 114 genomic DNA samples from healthy control participants were also genotyped for the rs13266634 SNP to examine the contribution of this SNP to susceptibility to type 1 diabetes in the Japanese population. This study was approved by the appropriate ethical committees and informed consent was obtained from all participants.
The ZnT8 complementary DNA (cDNA) constructs used in this study were the cytoplasmic carboxy-terminal domains (aa268-369) of human ZnT8 carrying either $325 \operatorname{Trp}(\mathrm{CW})$ or $325 \mathrm{Arg}(\mathrm{CR})$ and a fusion of the $\mathrm{CW}$ and CR with a CLFCEDPCDPSTPPGSSGGGKDFSILLME hinge junction generated by PCR. These cDNA were cloned into a pCDNA3.1 directional TOPO vector (Invitrogen, Carlsbad, CA, USA). The hybrid ZnT8 construct was designated as CW-CR.

Autoreactivity to ZnT8 CW, CR and CW-CR constructs was determined by radiobinding assay as described previously [2]. Positive control and negative control sera were included in each assay, and the antibody titres were expressed as an index defined as follows: (cpm in the unknown sample-cpm in the negative control)/(cpm in the positive control-cpm in the negative control). As a positive control for all constructs, a New Zealand white rabbit antiserum raised against an affinity-purified glutathione $S$ transferase fusion protein containing the cytoplasmic domain (aa268-369) of human ZnT8 was used. Classification of a sample as positive for ZnT8A was based on the 99th percentile of sera from 139 healthy control participants with no family history of diabetes. This corresponded to an index of 0.018 for $\mathrm{CW}$ autoantibodies, 0.016 for CR autoantibodies and 0.007 for $\mathrm{CW}-\mathrm{CR}$ autoantibodies, respectively. The inter- and intra-assay $\mathrm{CV}$ were $5.9 \%$ and $6.8 \%$ for $\mathrm{CW}$ autoantibodies, $10.4 \%$ and $5.7 \%$ for $\mathrm{CR}$ autoantibodies and 9.6\% and $4.6 \%$ for CW-CR autoantibodies, respectively.

The SLC30A8 rs13266634 C > T polymorphism was genotyped by PCR-RFLP. The PCR products were digested with a PvuII, resolved on a 3\% agarose gel and stained with ethidium bromide. No deviations from Hardy-Weinberg equilibrium were observed in control participants $(p=0.36)$. Statistical analyses were performed with the StatView 5.0 software (SAS Institute, Cary, NC, USA). The correlation between autoantibody titres was analysed using Spearman's rank-correlation test. Group comparisons of ZnT8A prevalence and titre were analysed by $\chi^{2}$ test and MannWhitney $U$ test, respectively. A value of $p<0.05$ was considered statistically significant.

\section{Results}

Of 270 patients with type 1 diabetes, 75 (28\%) were ranked as positive for $\mathrm{ZnT} 8 \mathrm{CW}-\mathrm{CR}$ autoantibodies, whereas autoantibodies to $\mathrm{CW}$ and $\mathrm{CR}$ constructs were positive in $37(14 \%)$ and $36(13 \%)$ patients, respectively. All sera positive for either $\mathrm{CW}$ or $\mathrm{CR}$ autoantibodies were positive for CW-CR autoantibodies. However, 20 (7\%) patients were CW-CR autoantibody-positive but negative for autoantibodies to $\mathrm{CW}$ and $\mathrm{CR}$. The prevalence of $\mathrm{CW}-\mathrm{CR}, \mathrm{CW}$ and CR autoantibodies in 112 new-onset patients (acute 
Fig. 1 Correlation between the reactivity to $\mathrm{ZnT} 8 \mathrm{CR}$ and $\mathrm{CW}$ construct (a) and the comparison between the titres of $\mathrm{ZnT} 8$ autoantibodies and SLC $30 A 8$ genotype $(\mathbf{b}, \mathbf{c})$ in Japanese patients with type 1 diabetes. a White circles, CC genotype; black circles, CT genotype; triangles, TT genotype. $\mathrm{CC}+\mathrm{CT}$ vs TT: $p<0.0001$ (b), $p=0.0024$ (c)

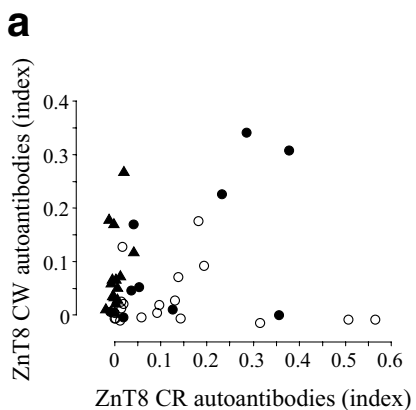

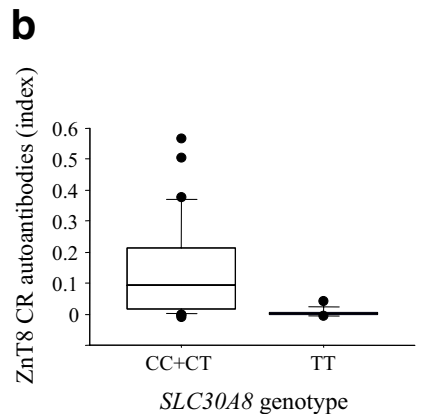

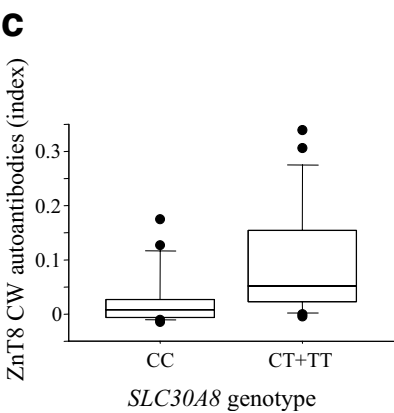

onset, slow onset, fulminant) was $36 \%, 16 \%$ and $20 \%$, respectively. CW-CR autoantibodies were more prevalent in younger $(\leq 20$ years $)$ new-onset patients compared with patients with age at onset of $>20$ years $(47 \%$ vs $28 \%$, $p<0.05)$. Among 171 patients with genomic DNA, 63 patients (37\%) were 325Arg (CC) homozygotes in SLC30A8, 36 (21\%) were homozygotes for the 325Trp (TT) and 72 $(42 \%)$ were CT heterozygotes. The allele frequency of rs13266634 in patients was not significantly different from that in the 114 healthy control participants (C allele: 57\% and T allele: $43 \%$, OR $1.02,95 \%$ CI $0.73-1.43$ ).

Forty-four patients were positive for CW-CR autoantibodies among the 171 patients with genomic DNA samples and they were used to examine the association between humoral autoreactivity to $\mathrm{ZnT} 8$ and the $S L C 30 A 8$ variant. Among 19 patients with the CC genotype eight patients $(42 \%)$ reacted with the $\mathrm{CR}$ construct alone, six patients (32\%) reacted with both $\mathrm{CR}$ and $\mathrm{CW}$ constructs, and only one patient $(5 \%)$ reacted with the $\mathrm{CW}$ construct alone. Conversely, 11 (73\%) of 15 patients with the TT genotype reacted with the $\mathrm{CW}$ construct alone and no one reacted with the CR construct alone. Nine of the ten patients (90\%) with the CT genotype reacted with either the $\mathrm{CR}$ or the $\mathrm{CW}$ construct. The prevalence of $\mathrm{CR}$ autoantibodies in patients carrying a $\mathrm{C}$ allele $(\mathrm{CC}+\mathrm{CT})$ was therefore significantly higher than that in TT homozygotes $(p<0.0001)$. In contrast, autoantibodies to the $\mathrm{CW}$ construct were more prevalent in patients with a $\mathrm{T}$ allele $(\mathrm{TT}+\mathrm{CT})$ than in those with the CC genotype $(p<0.01)$ (Table 1$)$. As shown in

Table 1 The prevalence of autoantibodies to the ZnT8 CR or CW construct in ZnT8 CW-CR autoantibody-positive patients with type 1 diabetes classified by SLC30A8 genotype

\begin{tabular}{lccr}
\hline SLC30A8 genotype & $n$ & \multicolumn{2}{c}{ Prevalence of autoantibodies to: } \\
\cline { 3 - 4 } & & CR & CW \\
\hline CC & 19 & $14(73.7)$ & $7(36.8)^{\mathrm{a}}$ \\
CT & 10 & $9(90.0)$ & $6(60.0)$ \\
TT & 15 & $2(13.3)^{\mathrm{b}}$ & $13(86.7)$ \\
\hline
\end{tabular}

Data are $n(\%)$

${ }^{\mathrm{a}} p<0.01$ vs $\mathrm{CT}+\mathrm{TT}$ genotype; ${ }^{\mathrm{b}} p<0.0001$ vs $\mathrm{CC}+\mathrm{CT}$ genotype

Fig. 1a, there was no overall correlation between titres of CW autoantibodies and CR autoantibodies $(R=0.069$, $p=0.66)$ and the autoreactivity to ZnT8 variant forms was closely associated with the $S L C 30 A 8$ genotype. The titre of $\mathrm{CR}$ autoantibodies in patients with a $\mathrm{C}$ allele was significantly higher than that in TT homozygotes $(0.139 \pm$ 0.158 vs $0.002 \pm 0.014, P<0.0001$ ) (Fig. 1b). In contrast, the titre of $\mathrm{CW}$ autoantibodies was significantly higher in patients with a $\mathrm{T}$ allele than in CC homozygotes $(0.093 \pm$ 0.103 vs $0.029 \pm 0.053, p=0.0024$ ) (Fig. 1c).

\section{Discussion}

This study has demonstrated that (1) the $S L C 30 A 8$ variant residue at aa325 is critically involved in the binding of ZnT8A; (2) the SLC30A8 genotype is an important determinant of autoantibody specificity; (3) the hybrid $\mathrm{CW}-\mathrm{CR}$ construct is a superior probe for the detection of ZnT8A; and (4) rs13266634 may not contribute to the genetic susceptibility to type 1 diabetes in the Japanese population. The first two points were recently proven in US patients [7]. However, the prevalence of $\mathrm{CW}$-specific autoantibodies in patients with either $\mathrm{CW}$ or CR autoantibodies was higher in this study $(35 \%$ vs $13 \%, p<0.0005)$ because of the difference in the frequency of TT homozygotes between Japanese and US populations. Currently, it is not known whether Arg325Trp SNP affects the functional properties of ZnT8. However, the amino acid chain that includes 325Arg has a protein kinase A and protein kinase $\mathrm{C}$ recognition motif (R-X-S/T) and rs13266634 SNP disrupts this motif, indicating the possible alteration of zinc transporter function. As differences in the epitope specificity of autoantibodies to GAD65 and insulinoma-associated antigen-2 are linked to the progression or the clinical heterogeneity of type 1 diabetes in Japanese patients [8,9], it is worth investigating the clinical role of $\mathrm{ZnT} 8 \mathrm{CW}$ and $\mathrm{CR}$ autoantibodies in the Japanese population.

Although the molecular mechanisms for the production of anti-islet autoantibodies in type 1 diabetes are largely unknown, the interactions of autoantibody with protein antigen usually depend on the conformational structure of 
the epitopes [10]. Tryptophan (W) encoded by TGG is a hydrophobic amino acid that tends to cluster on the inside of the protein to avoid contact with the aqueous environment and so it is unlikely to form the same bonding arrangement as the hydrophilic amino acid, arginine (R) encoded by $\mathrm{CGG}$. This may explain the observed differences in binding where the majority of ZnT8A in patients carrying the TT genotype did not recognise the CR construct. However, the findings that $37 \%$ of CC homozygous patients reacted with the CW construct and $13 \%$ of TT homozygous patients reacted with the CR construct indicate that the amino acids neighbouring aa325 are also involved in the ZnT8A binding.

The strength of this study is its use of a hybrid ZnT8 molecule (CW-CR) to identify ZnT8A-positive individuals; this allowed a $15-20 \%$ increase of sensitivity with the same specificity in our patients. Limitations are that about $60 \%$ of samples were obtained from long-standing cases and a minimum OR to obtain sufficient power $(>80 \%)$ under the assumption of $\alpha=0.05$ and observed allele frequency among controls was 1.9 in our case-control study.

In conclusion, the present study confirmed that a variant residue at aa325 is a key determinant of humoral autoreactivity to ZnT8 and that the SLC30A8 genotype is an important determinant of autoantibody specificity. Furthermore, the utility of the hybrid ZnT8 construct should be considered for ZnT8A screening in the relatives of patients with type 1 diabetes and in the general population.

Acknowledgement This study was partly supported by a grant from the Ministry of Education, Culture, Science, Sports and Technology of Japan.
Duality of interest The authors declare that there is no duality of interest associated with this manuscript.

\section{References}

1. Kambe T, Yamaguchi-Iwai Y, Sasaki R, Nagao M (2004) Overview of mammalian zinc transporters. Cell Mol Life Sci 61:49-68

2. Wenzlau JM, Juhl K, Yu L et al (2007) The cation efflux transporter ZnT8 (Slc30A8) is a major autoantigen in human type 1 diabetes. Proc Natl Acad Sci U S A 104:17040-17045

3. Chimienti F, Devergnas S, Favier A, Seve M (2004) Identification and cloning of a beta-cell-specific zinc transporter, ZnT-8, localized into insulin secretory granules. Diabetes 53:23302337

4. Sladek R, Rocheleau G, Rung J et al (2007) A genome-wide association study identifies novel risk loci for type 2 diabetes. Nature 445:881-885

5. Lu M, Fu D (2007) Structure of the zinc transporter YiiP. Science 317:1746-1748

6. The Expert Committee on the Diagnosis and Classification of Diabetes Mellitus (1997) Report of the Expert Committee on the Diagnosis and Classification of Diabetes Mellitus. Diabetes Care 20:1183-1197

7. Wenzlau JM, Liu Y, Yu L et al (2008) A common nonsynonymous single nucleotide polymorphism in the SLC30A8 gene determines ZnT8 autoantibody specificity in type 1 diabetes. Diabetes. doi:10.2337/db08-0522

8. Kawasaki E, Abiru N, Ide A et al (2003) Epitope analysis of GAD65 autoantibodies in Japanese patients with autoimmune diabetes. Ann N Y Acad Sci 1005:440-448

9. Kawasaki E, Sera Y, Fujita N et al (2001) Association between IA-2 autoantibody epitope specificities and age of onset in Japanese patients with autoimmune diabetes. J Autoimmun 17:323-331

10. Davies DR, Cohen GH (1996) Interactions of protein antigens with antibodies. Proc Natl Acad Sci U S A 93:7-12 